\title{
STORIA DELLA SCRITTURA E STORIA DEL LIBRO NELL'ANTICHITÀ GRECA E ROMANA MATERIALI PER UNO STUDIO
}

Un tema come quello di cui -a grandi (e superficiali?) linee- si deve qui trattare, richiede, prima ancora che si passi alla rassegna dei materiali, una distinzione non di comodo ma sostanziale tra le due «storie», quella della scrittura e quella del libro. Ed invero la storia della scrittura è storia delle forme grafiche nella totalità e diversità della loro realizzazione, tecnica e formale, ad opera degli scriventi, laddove invece la storia del libro manoscritto è storia delle sue componenti strutturali, tra cui v'è anche la scrittura, o meglio una serie di scritture che sono state adoperate (pure, soltanto) nella prassi libraria. Questo è, dunque, l'unico punto di intersezione tra le due storie, importantissimo per le incidenze reciproche ch'esso può determinare.

Iniziando dai materiali per lo studio della storia della scrittura nel mondo greco e romano, è ovvio trattare prima della scrittura greca. A tal riguardo, a venire immediatamente in considerazione sono i ritrovamenti greco-egizi, papiri e pergamene; ma questi possono documentare lo svolgimento delle forme grafiche solo a partire dalla fine del secolo IV a.C.: ai papiri da tempo noti cosiddetti «Persiani di Timoteo», "Maledizione di Artemisia» e documento di Elefantina del 311 a.C. (vd., per i primi due, M. Norsa, La scrittura letteraria greca dal secolo IV a.C. all'VIII d.C., Firenze 1939, tav. 1, e per il primo e il terzo W. Schubart, Papyri Graecae Berolinenses, Bonnae 1911, tavv. 1-2) si devono aggiungere un papiro scoperto a Saqqâra e databile intorno alla fine del terzo venticinquennio del IV secolo a.C. (E.G. Turner, $A$ Commanderin-Chief's from Saqqâra, in Journal of Egyptian Archaeology, 60, 1974, pp. 239-242) e un frammento orfico da Derveni in Macedonia, quest'ultimo riferibile alla fine del IV o all'inizio del III secolo a.C. (E.G. Turner, Greek Manuscripts of the Ancient World, Oxford 1971, tav. 51). Per l'epoca anteriore è necessario, invece, rifarsi alle testimonianze epigrafiche, le quali possono offrire solo una visione parziale della scrittura in uso nel mondo greco tra l' VIII e il $\mathrm{V}$ secolo a.C. Va sottolineato, piuttosto, che tali testimonianze epigrafiche non devono intendersi limitate alle manifestazioni più vistose, perché pubbliche $\mathrm{e}$ ufficiali, ma estendersi a tutta la cultura scritta recuperabile di quell' epoca: 
iscrizioni di possesso o di dedica-consacrazione reperibili su vasi, oggetti, cippi (M. Guarducci, Epigrafia greca, I, Caratteri e storia della disciplina. La scrittura greca dalle origini all'età imperiale, Roma 1967); scritte di contenuto sacrale su lamine auree (vd. almeno G. Zuntz, Persephone. Three Essays on Religion and Thought in Magna Grecia, Oxford 1971, e G. Foti-G. Pugliese Carratelli, Un sepolcro di Hipponion e un nuovo testo orfico, in La parola del passato, 29, 1974, pp.91-126; serie alfabetiche incise su tavolette bronzee o su altri oggetti, da ritenere tuttavia non d'uso scolastico ma di carattere magico-sacrale ( $\mathbf{M}$. Lejeune, Sur les abécédaires grecs archaiques, in "Revue de philologie», 57, 1983, pp. 7-12); scritture, sempre su bronzo, di contenuto vario (atti di donazione, lettere, formule magiche); firme di decoratori di vasi (H.R. Immerwahr, The Signatures of Pamphaios, in "American Journal of Archaeology", 88, 1984, pp. 341-352); graffiti, quali sono venuti alla luce nell' agorà ateniese (M. Lang, Graffiti in the Athenian Agora, Princeton 1974). Tra tali materiali va richiamata l'attenzione su di una letera incisa su lamina bronzea da Berezan, nel Ponto, databile al V secolo e assai interessante dal punto di vista grafico (B. Bravo, Une lettre de plomb de Berezan: colonisation et modes de contact dans le Pont, in Dialogues d'bistoire ancienne, 1, 1974, pp. 110-187). Resta negata per l'epoca anteriore al IV secolo a.C. una conoscenza diretta della scrittura adoperata nei libri, i quali sono da ritenere scarsamente diffusi fino a tutto il $\mathrm{V}$ secolo ca. (in una cultura di tipo orale, qual è quella della Grecia arcaica e della prima età classica, i libri sono destinati per lo più alla conservazione di determinate opere letterarie, non alla circolazione e alla lettura). Ugualmente negata resta per quest'epoca anche una conoscenza diretta della scrittura adoperata nei documenti scritti su materiali leggeri (tavolette o papiro ) conservati negli archivi delle poleis (E. Posner, Archives in the Ancient World, Cambridge [Mass.] 1972). Dai citati materiali del IV secolo, comunque, pare potersi desumere che non doveva esservi sostanziale divaricazione grafica tra scritture adoperate su supporti diversi e con funzioni diverse.

Con la fine del secolo IV a.C., s'è detto, ma soprattutto a partire dall'inizio del III, lo svolgimento della scrittura greca nella sua fase maiuscola si può seguire attraverso una serie assai congrua di testimonianze scritte che tra i secoli I e III d.C. diverranno abbondantissime. E'ovvio pensare immediatamente ai materiali, librari e documentari, restituiti dalla chora egizia e pubblicati nelle numerose raccolte papirologiche (una lista in E.G. Turner, Greek Papyri. An Introduction, Oxford 1968 [1980 $\left.{ }^{2}\right]$, pp. 154-171; ed. ital. Papiri greci, a cura di M. Manfredi, Roma 1984, pp. 179-212: tali materiali, infatti, constituiscono il cardine di ogni studio della scrittura greca dal IV secolo a.C. fino alla tarda antichità (cataloghi di papiri: vd. per i letterari R.A. Pack, The Greek and Latin Literary Texts from Greco-Roman Egypt, Ann Arbor 1965'; per i biblici e cristiani, K. Aland, Repertorium der griechischen christlichen Papyri, I, Biblische 
Papyri, Berlin-New York 1976, e J. van Haelst, Catalogue des papyrus littéraires juifs et chrétiens, Paris 1976; per i documentari Sammelbuch griechischer Urkunden aus Aegypten, raccolta iniziata da F. Preisigke nel 1915 e continuata da F. Bilabel, E. Kiessling, H.-A. Rupprecht; inoltre, in mancanza di raccolte sistematiche di papiri greci riprodotti, vd. almeno le scelte -alcune già ricordate- di M. Norsa, La scrittura letteraria greca dal IV secolo a.C. all'VIII d.C., Firenze 1939, e Papiri greci delle collezioni italiane, voll. I-III, Roma 19291946, R. Seider, Palaeographie der griechischen Papyri, voll. I-II, Stuttgart 1967-1970, E.G. Turner, Greek Manuscripts of the Ancient World, Oxford 1971, G. Cavallo and H. Maehler, Greek Bookbands of Early Byzantine Period [A.D. 400-800], London 1986). Ma -a differenza di quanto è avvenuto per la storia della scrittura latina, come si vedrà -proprio l'abbondanza di tali materiali greci (frammenti più o meno ampi di papiro o pergamena) ha fatto sì che gli studi di storia della scrittura greca abbiano finito con il trascurare testimonianze scritte sia di altra origine (pur se scarse, ma non meno significative: vd. G. Cavallo, Unità e particolarismo grafico nella scrittura greca dei papiri, in Proceedings of the Twelfth International Congress of Papyrology, Toronto 1970, pp. 77-83) sia di altra tipologia, greco-egizie e non. Per quanto riguarda le scritture d'uso librario, vi sono frammenti originari della Macedonia (il già ricordato papiro di Derveni), della Mesopotamia (da Dura Europos), della Palestina (da Murabba' ât, Qumrân, Nessana), della Nubia (da Qasr Ibrîm: vd. J.M. Plumley, The Christian Period at Qasr Ibrim. Some Notes on the MSS Finds, in Nubia. Récentes recherches, réd. K. Michalowski, Varsovie 1975, pp. 101-107; C.H. Roberts- J.M. Plumley, An Uncial Text of St. Mark in Greek from Nubia, in "Journal of Theological Studies», N.S., 27, 1976, pp. 34-45; E.G. Turner-M.E. Weinstein, Greek and Latin Papyri from Qasr Ibrim, in "Journal of Egyptian Archaelogy", 62, 1976, pp. 115-130; W.H.C. Frend, The Greek Liturgical Papyri from the Cathedral of Qasr Ibrim, in "Études nubiennes», Cairo 1978, p. 95, e Some Greek Liturgical Fragments from Qasr Ibrim in Nubia, in "Studia Patristica», XV, Berlin 1984, pp. 545-553), del Sinai (L. Politis, Nouveaux manuscrits grecs découverts au Mont Sinai. Rapport préliminaire, in "Scriptorium», 34, 1980, pp. 5-17); ma il fondo più consistente viene da Ercolano, le cui scritture più antiche si devono ritenere prodotte in àmbito greco-orientale ed offrono perciò un quadro articolato delle forme greche d'uso librario tra il III e il I secolo a.C., mentre, d'altro canto, le scritture più recenti, adoperate per trascrivere le opere di Filodemo di Gadara, sono sicuramente databili tra la tarda età repubblicana e il 79 d.C., sicché permettono di agganciare ad esse tutta una serie di scritture di assai incerta datazione (G. Cavallo, Libri scritture scribi a Ercolano, Napoli 1983). Quelche parola in più merita, tra i materiali recentemente ritrovati ad Ai Khanoum in Afghanistan, un "pezzo» di indole particolare: si tratta dell'impronta lasciata a terra da 
un frammento di papiro di contenuto filosofico; impronta nella quale ovviamente la scrittura si presenta in posizione inversa, sicché può essere letta o con l'aiuto di uno specchio o -come è stato fatto- traendo una fotografia da un negativo posto a sua volta in posizione inversa in modo che ne risulti normale la scrittura della stampa. Il "pezzo» è in corso di studio ad opera di Claude Rapin dell'Università di Losanna, che propone di assegnarlo al III secolo a.C.

Ancor più numerose si mostrano le testimonianze non egizie per quanto riguarda le scritture d'uso documentario. Dalla stessa Palestina proviene tutta una serie di documenti dislocati nel tempo (dall'Ammonitide, ed ancora da Murabba'ât e Nessana); e vi sono inoltre documenti originari del Kurdistan (da Avroman), della Siria, Pamfilia, Licia, Fenicia, Bitinia e della Nubia (per quest'ultima vd. Turner-Weinstein, Greek and Latin Papyri cit., pp. 115-130, e T. Hägg, Nubicograeca I-III. Bemerkungen zu griechischen Texten aus Nubien, in "Zeitschrift für Papyrologie und Epigraphik», 54, 1984, pp. 101-112); né manca qualche pezzo dall'Arabia, (da Nahal Hever: N. Lewis, Two Greek Documents from Provincia Arabia, in «Illinois Classical Studies», 3, 1978, pp. 100-114). I documenti più numerosi provengono dalla Mesopotamia, da Dura Europos, coprendo un arco cronologico assai ampio, dal III secolo a.C. al III d.C. Vi sono inoltre - di origine sia egiziana sia non egiziana- materiali, letterari e documentari, non ancora indagati fino in fondo (o non indagati sotto lo especifico angolo visuale della storia della scrittura): $\mathrm{mi}$ riferisco alle scritte su tavolette lignee cerate plumbee, o a quelle su ostraca e frammenti di terracotta di vario genere (qui vorrei ricordare almeno la piccola, ma utile raccolta di P. Mertens, Les ostraca littéraires grecs, in "Orientalia Lovaniensia Periodica», 6-7, 1975-1976, pp.397-409, o ancora -assai interessanti perché di età ellenistica e prodotte in Afghanistan-Les inscriptions économiques de la trésorerie bellénistique d'Ai Kbanoum, ultimamente edite da C. Rapin, in «Bulletin de Correspondance Hellénique», 107, 1983, pp. 315-371), o ai graffiti (la recente pubblicazione di D. Devanchelle-G. Wagner, Les graffites au Gebel Teir. Textes démotiques et grecs, Le Caire 1984, offre una serie di testimonianze di notevole valore).

Tali materiali epigrafici, qualunque ne sia l'origine, devono essere recuperati anch'essi alla storia della scrittura greca, con la coscienza, tuttavia, del carattere specifico di quei materiali e dell'autonomia scientifica della disciplina che ne fa oggetto di indagine. Ed invero -nonostante una importante inchiesta pubblicata da "Scrittura e civiltà», 5(1981), pp. 265-312, sui rapporti tra paleografia ed epigrafia- la problematica in tal senso resta aperta sia sul piano metodologico (la diversità delle tecniche rende impraticabile un approccio alle epigrafi con i criteri di valutazione paleografica applicati alle scritture su papiro/pergamena) sia sul piano delle interazioni specifiche tra i due mondi di scrittura (i quali restano sostanzialmente separati, a parte fenomeni particolari 
del tutto sovrastrutturali). Resta il fatto -sottolineato negli interventi contenuti in Scrittura e civiltà - che paleografia ed epigrafia sono ambedue scienze della comunicazione scritta, che le testimonianze oggetto di studio hanno un comune referente negli ambienti culturali e sociali in cui sono state prodotte e utilizzate, che l'una e l'altra possono offrire un terreno di indagine, reciprocamente complementare, allo studio delle abbreviazioni e dei sistemi di punteggiatura; ma tutto questo non è ancora sufficiente. Quel che resta positivo è che il discorso è ormai avviato, e certo non mancherà di provocare approfondimenti ulteriori.

In conclusione, una storia della scrittura greca nella sua fase maiuscola, pur conservando ovviamente il suo cardine nei «papiri» greco-egizi (sui quali perciò non mi è parso necessario soffermarmi a lungo) non può prescindere da testimonianze d'altra indole e d'altra origine se vuol dare una visione del fenomeno grafico articolato per tecniche di esecuzione, modi d'uso della scrittura particolaristici o unitari nel tempo e nello spazio, livelli sociali di fruizione, funzioni diverse della scrittura stessa.

Diversamente dalla storia della scrittura greca, la storia della scrittura latina non dispone, a suo fondamento, di una messe di materiali papiracei e membranacei altrettanto cospicua; ed è per questo che l'utilizzazione di altri documenti (iscrizioni varie, tavolette, graffiti) è stata -da parte dei paleografi latini- assai più vasta (ma non sistematica). Fino alle soglie dell'età augustea, per la storia della scrittura latina, non si dispone comunque di altro. Tra i materiali di età più antica è d'obbligo ricordare la fibula Praenestina, riferita ad una data intorno al 600 a.C., ma la cui autenticità è stata di recente messa fortemente in dubbio (Vd. M. Guarducci, La cosiddetta Fibula Praenestina. Antiquari, eruditi e falsari nella Roma dell'Ottocento, in Memorie dell'Accademia Nazionale dei Lincei, Cls. di scienze morali, storiche e filologiche, ser. VIII, 24, 1980, pp. 413-574, e 28, 1984, pp. 127-177). Ad un arco di tempo tra la fine del VI secolo e la fine del V secolo a. C. vanno riferiti il lapis niger, la lamina bronzea di Lavinio, il vaso di Duenos. Sempre per lo stesso periodo, inoltre, non mancano altre iscrizioni strumentali (vd. G. Colonna, La diffusione della scrittura, in Civiltà del Lazio primitivo, Roma 1976, pp. 372-376, e Le iscrizioni strumentali latine del VI e V secolo a.C., pubbl. come appendice al vol. $\mathrm{V}$ degli Scripta minora degli Studi Archeologici dell'Istituto Olandese a Roma, s.d., pp.41-690).

Anche per l'età republicana i materiali non sono molti; a parte alcune scoperte più recenti (ma non in quantità cospicua), tali materiali -inscrizioni su cippi, olle, buccheri e altri oggetti- risultano raccolti e indagati da Giorgio Cencetti in relazione al filone corsivo (Ricerche sulla scrittura latina nell'età arcaica, I, Il filone corsivo, in «Bullettino dell'Archivio Paleografico Italiano», 
n.s., 2-3, 1956-1957, I, pp. 175-205), mentre restano da indagare meglio le testimonianze epigrafiche di carattere pubblico e ufficiale, quelle, per intendersi, ove si assiste al processo di formazione e stilizzazione della capitale epigrafica. Per quest'epoca mancano materiali da cui attingere una qualche, sia pur limitata conoscenza, della scrittura adoperata nei libri o in documenti di papiro; tuttavia, se P. Berol. 13956, una lettera su papiro, va datato intorno alla metà del I secolo a.C., esso costituisce uno dei più antichi documenti del genere ed attesta una capitale già formata.

E'a partire, in pratica, dall'età augustea che la documentazione si fa più fitta, divenendo sempre più abbondante e culminando tra il I e il III secolo d.C. Si dispone di inscrizioni di carattere ufficiale, manifesti a pennello, scritte su tavolette di varia indole, ostra ca, graffiti su pareti o terracotta; ma ormai si dispone pure di una larga documentazioni di scritture su papiro, sia librarie sia documentarie, a quanto mostrano le grandi raccolte dei Codices Latini Antiquiores di E.A. Lowe (ormai completi: voll. I-XI e Suppl., Oxford 1934-1971; ulteriore supplemento dovuto a B. Bischoff-V. Brown, Addenda to Codices Latini Antiquiores, in «Mediaeval Studies», 47, 1985, pp. 317-366) e delle Chartae latinae Antiquiores (ancora incomplete: sono stati finora pubblicati, a cura di A. Bruckner, R. Marischal, J.-O. Tjäder, A. Petrucci, J. Vezin e altri, i voll. I-XV, XVII-XVIII, XX-XXIV, Olten-Lausanne e Dietikon-Zürich 1954-1985). La raccolta dei Codices Latini Antiquiores offre in pratica tutti i materiali librari; ai quali va aggiunto solo un certo numero di papiri latini di Ercolano che attende di essere indagato adeguatamente e che può offrire alcune novità (vd. Appendice a questa rassegna). Per quanto concerne le scritture di uso non-librario, va sottolineato l'interesse dei manifesti a pennello, elettorali o d'altro genere (per es. gli annunci di spettacoli gladiatori: vd. P. Sabbatini Tumolesi, Gladiatorum paria. Annuci di spettacoli gladiatori a Pompei, Roma 1980); vi sono poi le scritte su tavolette, tra le quali vanno ricordate almeno quelle pompeiane dell'archivio di Lucio Cecilio Giocondo e daciche trovate in Transilvania, le numerose -molte ancora inedite- tavolette ercolanesi, ed ancora quelle di Vindonissa in Svizzera e di Vindolanda in Britannia: (su queste ultime vi è il recente studio di A.K. Bowman-J.D. Thomas, Vindolanda: the latin Writing-Tablets, London 1983, lavoro nel quale si trova anche tutta la bibliografia sugli altri gruppi di tavolette qui ricordati). Si tratta di materiali riferibili ai primi tre secoli dell'impero; di data, invece, più tarda è il gruppo delle cosiddette "Tablettes Albertini» proveniente da un sito tra Algeria e Tunisia (Tablettes Albertini. Actes privés de l'époque vandale Ifin $d u V^{\prime}$ siècle], a cura di C. Courtois, L. Leschi, Ch. Perrat, Ch. Saumagne, Paris 1952). Tavolette di varie epoche sono state restituite anche dall'Egitto. Non molto conto si è tenuto finora delle scritture su ostraca, le quali possono essere adeguatamente indagate nei materiali provenienti dal Wâdi Fawâkhir in 
Egitto (O. Guéraud, Ostraca grecs et latins de l'Wâdi Fawakkbir, in «Bulletin de l'Institut d'Archéologie Orientale», 41, 1942, pp. 141-196. in part. pp. 153-161), ma soprattutto nei ritrovamenti, assai numerosi, di Bu Njem in Libia (R. Rebuffat-R. Marichal, Les ostraca de Bu Njem, in «Revue des études latines», 51, 1973, pp. 281-286; R. Marichal, Les ostraca de Bu Njem, en Comptes Rendus de l'Académie des Inscriptions et Belles Lettres, 1979, pp. 436452). Di grandissima importanza per una conoscenza della scrittura latina in età romana risultano anche i graffiti, tra i quali mi limito a ricordare $\mathrm{i}$ gruppi più consistenti: quelli di Roma, Ostia, Ercolano e Pompei in Italia (Vd. H. Solin, Graffiti del Palatino, I, Paedagogium, Helsinki 1966; P. Castrén-H. Lilius, Graffiti del Palatino, II, Domus Tiberiana, Helsinki 1970; ed ancora, dello stesso $\mathrm{H}$. Solin, Die herkulanensischen Wandinschriften. Ein soziologischer Versuch, in "Cronache Ercolanesi», 3, 1973, pp. 97-103. Die Wandinschriften im sog. Haus des M. Fabius Rufus, in Neue Forscbunger in Pompeji, hrsg. v. B. Andreae u. H. Kyrieleis, Rechlinghausen 1975, pp. 243-266, Le iscrizioni parietali, in «Pompei», 79, Napoli 1984, pp. 278-288); quelli di La Graufesenque/Condatomagos in Gallia (F. Hermet, La Graufesenque/Condatomago, I, Vases sigillés; II, Graffites, Paris 1934; A. Albenque, Nouveaux graffites de la Graufesenque, in "Revue des études anciennes», 53, 1951, pp. 71-81, A. Aymard, Nouveaux graffites de La Graufesenque, II, ibid., 54, 1952, pp. 93-101, e III, ibid., 55 (1953), pp. 126-131; R. Marichal, Nouveaux graffites de La Graufesenque, IV, ibid., 76, 1974, pp. 85-110 e 266-292; vd. anche A. Petrucci, Per la storia della scrittura romana: i graffiti di Condatomagos, in «Bullettino dell' Archivio Paleografico Italiano», 3 " ser., 1, 1962, pp. 85-132); quelli di Magdalensberg in Carinzia (R. Egger, Die Stadt auf dem Magdalensberg. Ein Grosshandel platz. Die ältesten Aufzeichnungen des Metallwarenhandels auf dem Boden Oesterreichs, Wien 1961); quelli di Haltern in Renania (B. Galsterer, Die Graffiti auf der römischen Gefasskeramik aus Haltern, Münster 1983); quelli di Usk in Gran Bretagna (M.W.C. Hassal, Inscriptions and Graffiti, in G.C. Boon - M.W.C. Hassall, Report on the Excavation at Usk 1965-1976. The Coins, Inscriptions and Graffiti, Cardiff 1982, pp. 45-61). Non mancano, infine, tavolette plumbee di carattere magico-sacrale interessanti sotto l'aspetto grafico (vd., per es., W.S. Fox, The Jobns Hopkins Tabellae defixionum, Baltimore 1912, e ultimamente, M. Lejeune, avec L. Fleuriot, P.-Y. Lambert, R. Marichal, A. Vernhet, Le plomb magique du Larzac et les sorcières gauloises, Paris 1985, con studio paleografico alle pp. 10-24).

L'esclusione -lamentata da Armando Petrucci (vd. Gnomon, 51, 1979, P. 27)- dei materiali quali tavolette lignee e cerate, ostraca tabelle plumbee dalle Chartae Latinee Antiquiores rende e continuerà a rendere disorganicamente reperibili testimonianze scritte che contribuiscono tutte alla conoscensa della scrittura latina d'uso non librario in età romana. Meglio noti dunque risultano -grazie alle stesse Chartae Latinae Antiquiores- i documenti su papiro, i quali 
tuttavia, poiché quasi totalmente di origine amministrativa civile o militare, rischiano di dare una visione riduttiva del fenomeno grafico. Sicché, se tali materiali possono portare a osservazioni o conclusioni di peso per quanto concerne uso, funzioni, gerarchie delle scritture burocratiche (basti ricordare i lavori di G. Cencetti, Note paleografiche sulla scrittura dei papiri latini dal I al III secolo d.C., in Memorie dell'Accademia delle Scienze di Bologna, Classe di Scienze morali, 5, 1, 1950, pp. 2-58, e Paleografia e papirologia, in Guida allo studio della civiltà romana antica, II, Napoli 1961, pp. 595-688), quegli stessi materiali non devono essere utilizzati da soli quando si vogliano proporre tesi di carattere più generale. E' il caso di un lavoro, pur assai importante, di Emanuele Casamassima ed Elena Staraz basato esclusivamente su materiale papiraceo e inteso a spiegare il formarsi e l'affermarsi di un sistema grafico minuscolo (E. Casamassima-E. Staraz, Varianti e cambio grafico nella scrittura dei papiri latini. Note paleografiche, in «Scrittura e civiltà», 1, 1977, pp. 11-110). Qui si tocca uno dei nodi cruciali nella storia della scrittura latina, quello del passaggio dalla maiuscola alla minuscola o -se si preferisce seguire la scuola francese-dalla scrittura comune antica alla nuova. Ma una riproposizione di tutta la problematica, dopo la classica Paléographie romaine di Jean Mallon, pubblicata a Madrid nel 1952, deve tener conto di tutti i materiali disponibili individuandone uso e funzioni come prodotti scritti. In ultima analisi, e più in generale, anche per la storia della scrittura latina come per quella della scrittura greca, la strada da percorrere resta quella di una paleografia "totale», che interpreti e coordini in un discorso storico-critico unitario testimonianze scritte d'indole diversa, sincroniche o diacroniche, di uguale origine o dislocate.

Passando a considerare la storia del libro in età antica, va evitata -a differenza di quanto s'è fatto per la storia della scrittura- una distinzione tra mondo greco e mondo latino, in modo che meglio si possano cogliere analogie e differenze. Fino all'ultimo scorcio del IV secolo a.C. la nostra conoscenza del libro antico è soltanto indiretta, fondata su testimonianze letterarie (sovente peraltro assai frammentarie) o iconografiche, soprattutto vascolari (vd. almeno, per le prime G.F. Nieddu, Testo, scrittura, libro nella Grecia arcaica e classica: note e osservazioni sulla prosa scientifico-filosofica, in "Scrittura e civiltà», 8, 1984, pp. 213-261; per le seconde F.A.G. Beck, Album of Greek Education. The Greeks at School and at Play, Sidney 1975, ma non tralasciando di far qualche volta ricorso, pure, ai lavori di H.R. Immerwahr, Book Rolls on Attic Vases, in Classical Mediaeval and Renaissance Studies in Honour of B.L. Ullman, Roma 1964, pp. 17-48, e More Book Rolls on Attic Vases, in "Antike Kunst», 16, 1973, pp. 143-147). Esse valgono a far conoscere in qualche modo la diffusione sociale del libro -commisurata soprattutto sul rapporto 
oralità/cultura scritta- ma servono molto meno a dare una idea sufficientemente concreta della structtura materiale del libro stesso (una sintesi sulla diffusione del libro in quest'epoca, ma anche più tardi, risulta tracciata da B.M.W. Knox-P.E. Easterling, Books and Readers in the Greek World, in The Cambridge History of Classical Literature, I, Greek Literature, Cambridge 1985, pp. 1-41). Almeno fino al VI secolo, tuttavia, sembra che i testi letterari furono fissati su supporti scrittori duri (pelli, piombo, tavolette); si trattava dunque di libri (se è lecito adoperare questo termine) destinati ad una conservazione del papiro, in concomitanza con tutta una serie di fattori socioculturali, avviò un processo di strutturazione del libro in forme nuove, di cui noi conosciamo esiti ed ulteriori momenti evolutivi a partire dalla fine del IV secolo a.C. e soprattutto dal III (per i libri in età pre-alessandrina vd. il classico contributo di E.G.Turner, Athenian Books in the Fifth and Fourth Century, London $\left.1977^{2}\right)$.

Questo vale per il mondo greco; mentre per il mondo romano è necessario giungere al I secolo a.C. e ai papiri latini di Ercolano nonché a qualche fortunato ritrovamento d'altra origine per conoscere in concreto i primi libri di Roma antica: per l'età anteriore si hanno solo testimonianze indirette e peraltro assai scarse. I libri Sybillini, i primi libri che la tradizione ponga in mani romane, introdotti da Cuma a Roma all'epoca di Tarquinio il Superbo, erano scritti in greco. Si a notizia anche di libri lintei di contenuto sacrale, ma nessuna testimonianza si è direttamente conservata; e tuttavia, della strutturazione di tali libri si può forse avere un'idea abbastanza precisa dalla documentazione etrusca: il liber linteus di Zagabria, redatto in etrusco e di contenuto sacrale, si mostra di una lunghezza superstite di $\mathrm{cm} 340$ e di un'altezza di $\mathrm{cm} 35$, ma -fatto significativo- era ripiegato «a soffietto» e connotato da una «mise en page» riquadrata da filettatura in rosso, sicché le singole superfici di testo presentano figura assai simile alla pagina di un codice (vd. quanto scrive $\mathrm{F}$. Roncalli nel catalogo della mostra, allestita a Perugia, Scrivere etrusco. Dalla leggenda alla conoscenza. Scrittura e letteratura nei massimi documenti della lingua etrusca, Milano 1985, pp. 22-24). La stessa struttura fisica dovevano avere i libri lintei romani. Per quanto concerne i testi letterari, la documentazione diretta, s'è detto, inizia dalla fine del IV secolo a.C. per il mondo greco a dalla fine del I a.C. per quello romano; e tale documentazione direttamente conservatasi può essere meglio interpretata (e talora integrata) con la messe sia di testimonianze indirette reperibili in autori greci e latini sia di materiali iconografici (si possono ricordare alcuni studi non sempre recenti, comunque ancora utili: per le prime T. Birt, Das antike Buchwesen, Berlin 1882, e E.J. Kenney, Books and Readers in the Roman World, in The Cambridge History of Classical Literature, II, Latin Literature ed. by E.J. Kenney and W.V. Clausen, Cambridge 1982, pp. 3-32; per i secondi T. Birt, Die Bucbrolle in der Kunst, 
Leipzig 1907, e H.-I. Marrou, Mousikos aner. Étude sur les scènes de la vie intellectuelle figurant sur les monuments funéraires romains, Grenoble 1938). La documentazione libraria diretta viene quasi tutta dall'Egitto (rimando, anche per le eccezioni, ai materiali citati per la storia della scritiura), consentendo comunque, ove ben conservata, una conoscenza approfondita almeno del libro greco (resta fondamentale al riguardo il lavoro di W. Schubart, Das Busch bei den Griechen und Römern, Berlin-Leipzig $1921^{2}$ ). Il rotolo librario sembra si sia strutturalmente definito nel mondo greco in epoca alessandrina -lunghezza tra i m 2,50 e 12 ca., altezza tra i cm 16 e 24 ca., altezza delle colonne tra i cm 15-18, larghezza delle medesime tra i cm 4-8- subendo alcune variazioni nel corso del tempo; in particolare, in età romana risultano spesso accresciute sia l'altezza del rotolo sia le misure delle colonne (su questi ed altri aspetti dell' antico volumen vd. E.G. Turner, The Terms Recto and Verso. The Anatomy of the Papyrus Roll, Bruxelles 1978, T.C. Skeat, The Lenght of the Standard Papyrus Roll and the Cost-advantage of the Codex, in «Zeitschrift für Papyrologie und Epigraphik», 45, 1982, pp. 169-175, e Cavallo, Libri scritture scribi cit., pp. 14-27). Ma desumere per i rotoli latini più antichi una strutturazione uguale a quella dei greci può essere rischioso. A partire, comunque, dal I secolo a.C./I secolo d.C., i materiali raccolti nei Codices Latini Antiquiores e qualche papiro di Ercolano ancora inedito mostrano alcune caratteristiche individuabili: per es., in P. Herc. 831, il carmen de bello Actiaco databile tra il 31 a.C. e il 79 d.C., l'ampiezza delle colonne misura cm. 17; ugualmente in papiri letterari latini provenienti dall'Egitto le colonne si mostrano assai larghe, tra i cm 18 e i 24. Per quanto concerne un recente ritrovamento, il cosiddetto "papiro di Cornelio Gallo", la questione si presenta assai delicata (il papiro è stato edito da R.D. Anderson-P.J. Parsons-R.G.M. Nisbet, Elegiacs by Gallus from Qasr Ibrîm, in "Journal of Roman Studies», 69, 1979, pp. 125-155). Il "pezzo» offre una serie di caratteristiche nuove rispetto a quanto finora si conosceva sulla «impaginazione» del libro antico, ma essendo stato avanzato il sospetto che si tratti di un falso (vd. F. Brunhölzl, Der sogenannte Galluspapyrus von Qasr Ibrîm, in "Codices manuscripti», 10, 1984, pp. 33-37), è opportuno per il momento sospendere il giudizio.

E vengo ad uno dei nodi cruciali nella storia del libro antico: la sostituzione del codice al rotolo. I materiali relativi ai codici più antichi (ma anche tardoantichi fino al VI-VII secolo) si trovano raccolti e presentati criticamente nel magistrale volume di Eric G. Turner, The Tipology of the Early Codex, University of Pennsylvania 1977: anche se l'intento dell'autore è quello di studiare la struttura fisica del codice primitivo, non il cosiddetto "passaggio» dal rotolo al codice, non v'è dubbio che la raccolta porta un contributo fondamentale a tal problematica. Specificamente a quest'ultima, invece, hanno di recente dedicato un nuovo studio C.H. Roberts e T.C. Skeat, The Birth of 
the Codex, London-Oxford 1983; ma non è mio intento esporre in questa sede le tesi dei due studiosi e quindi contrapporvi le mie (il che ho avuto già occasione di fare in Studi italiani di filologia classica, 3. ${ }^{2}$ ser., 3, 1985, pp. 118-121): mi limiterò a proporre soltanto qualche riflessione.

Il dualismo rotolo/codice, ove rapportato al contesto complessivo della cultura scritta nel mondo antico, perde molto del suo valore e rende meno vistosa la cosiddetta "nascita del codice». Si deve tener conto, infatti, che le nostre conoscenze si fondano in sostanza sulla documentazione greco-egizia, la quale restituisce un'immagine fortemente univoca dei fenomeni sia per il fatto stesso ch'è la sola disponibile in quantità notevole, sia perché i prodotti scritti risultano strutturalmente condizionati dai modi di fabbricazione e di diffusione commerciale del papiro, materia scrittoria locale e perciò adoperata per qualsiasi tipologia di manufatto assai più largamente che altrove, sia infine per l'àmbito esclusivamente (o quasi) eccentrico dal quale quella documentazione è originaria. Ed invece nel mondo mediterraneo greco e romano la realtà doveva essere assai articolata; di certo non mancavano, con funzioni diverse da quelle del rotolo, forme alternative di produzione scritta: si conservano in misura non scarsa, s'è visto, tavolette cerate (e non), ostraca, lamine auree e plumbee, foglietti di vario tipo; ma sul fondamento di più sporadici e frammentari ritrovamenti o di fonti letterarie si può ricostruire un complesso di prodotti scritti molto più diversificato di quanto comunemente si creda: pugillares, in sostanza «note-books» in forma di codice; libelli, per lo più volantini in versi o in prosa affissi o distribuiti in luogo pubblico a scopo polemico e diffamatorio, ma che dovevano essere altrimenti strutturati (a quel che sembra proprio in forma di codice) se di contenuto più ampio; tabellae di tiglio assai sottili di solito riunite insieme in varia misura e conformazione. Ed invero, a quest'ultimo proposito, a Vindolanda, in Britannia, è documentata una forma di manufatto «a soffietto» composto di tavolette che nel mondo antico forse non era limitata solo alla prassi documentale e all'uso di materiale ligneo (sulle tavolette di Vindolanda rimando al già ricordato lavoro di Bowman e Thomas, spec. pp. 32-45). Tale forma è stata ritenuta tipologicamente lontana dal codice in quanto la scrittura vi figura su una sola facciata; ma -senza voler inferire alcuna diretta discendenza dell'una forma dall'altra, giacché la realtà sembra essere stata la coesistenza di prodotti scritti diversi- va almeno notato che anche certi codici rudimentali, veri e propri «note-books», quali P. Lit. Lond. 5+182 o, se il frammento è originario di un codice, P. Berol. 10569, riferibili al III secolo ca. e certo d'uso scolastico (l'uno è di contenuto miscellaneo, l'altro, di pergamena, reca versi omerici), risultano scritti su una sola facciata. La stessa caratteristica, inoltre, s' incontra in un vero e proprio codice letterario di ragguardevole formato, il cosiddetto Omero di Harris, frammento iliadico da assegnare al tardo II secolo piuttosto che alla fine del III come finora 
si è sempre ritenuto. Nel mondo antico è testimoniata, insomma, tutta una serie di prodotti scritti diversa dal rotolo giacché non legati a contenuti, convenzioni, usi di quest'ultimo; e tra i manufatti alternativi di questa specie vi era, e tale sostanzialmente rimase, almeno fino a tutto il III secolo, anche il codice, pur se in modi o materiali che per un'epoca anteriore al I-II le testimonianze lasciano solo fugacemente intravedere. Che poi venisse adottato sempre più e alla fine esclusivamente il codice dipese dal fatto che all'inicirca dall'età costantiniana si assiste ad una graduale omologazione di tipologie diverse di produzione libraria (e documentaria) in favore di quell' unica forma; omologazione dovuta, tra l'altro, ad un alfabetismo non più tanto diffuso da indurre l'esigenza di prodotti scritti differenziati per livelli sociali o per funzioni, ad una scuola che va riorganizzandosi e che istituzionalizza per gli autori adottati la forma del codice già da tempo in uso per $i$ «quaderni» di esercizi, ad un pubblico di nuovi ceti dirigenti educati sovente alle discipline tecniche e i più ormai cristiani che finiscono con l'imporre una structura libraria una volta prevalentemente destinata, proprio, ai testi di carattere pratico e al messaggio cristiano. Ma in ogni caso la trascrizione di opere antiche nella tipologia libraria del codice fu un fatto non programmatico, non organizzato, non sistematico; non vi furono in alcun momento determinate scelte o selezioni di quel che doveva essere trascritto dall'una all'altra forma. Si trattò, invece, di un processo lento e dilatato nel secoli, nel corso del quale un testo, convivendo a lungo i due tipi di libro, poté passare da rotoli a codici più volte, in tempi e luoghi diversi, secondo modalità varie, o anche potè esser trascritto nella nuova forma libraria una sola volta ed in un solo àmbito (con una gamma di possibilità molto vasta tra i due estremi...).

Un problema che trascende le stesse trasformazioni del libro è quello, assai complesso, del manoscrito - talora vero e proprio brogliaccio- d'autore quale poteva presentarsi strutturato nel mondo greco e romano; ma sembra che la stesura di un testo letterario sia avvenuta per lo più su tavolette lignee o cerate $\mathrm{e}$ qualche volta anche su rotolo (vd. G. Cavallo, I rotoli di Ercolano come prodotti scritti. Quattro riflessioni, in "Scrittura e civiltà», 8, 1984, pp. 12-20, e A. Locher-R.C.A. Rottländer, Überlegungen zur Entstebungsgeschichte der Naturalis Historia des älteren Plinius und die Schrifttäfelchen von Vindolanda, in Lebendige Altertumswissenschaft. Festgabe zur Vollendung des 70. Lebensjabres von $H$. Vetters..., Wien 1985, pp. 140-147). Resta da chiedersi se tavolette e rotoli costituissero modi alternativi per stesure d'autore o se rappresentassero fasi diverse e successive nella composizione di un'opera letteraria. Una risposta, in quanto fortemente problematica, è da lasciare in sospeso; peraltro, nella produzione testuale antica, le fasi attraverso cui quest'ultima passava, possono non essere state sempre le stesse qualitativamente e quantitativamente: a tipologie diverse di testo non si può escludere corrispondessero forme di elaborazione in qualche modo differenziate del discorso scritto. 
Nella mia trattazione si sarà notata l'assenza di due problematiche, le quali tuttavia sono state sempre sullo sfondo: da una parte la problematica inerente a quella che Armando Petrucci chiama «difusione sociale della scrittura", vale a dire l'alfabetismo a vari livelli e nelle sue varie realizzazioni e fruizioni pratiche; dall'altra la problematica inerente all'indole specifica dei testi veicolati dalla scrittura. Si tratta di due problematiche fondamentali, che continuamente interagiscono nella storia della scrittura, del libro e... del documento. Nel mondo greco il passaggio da una società alfabetizzata e colta quale quella dell' Atene di età classica ad una società paradossalmente analfabeta ed erudita come quella ellenistica, e nel contempo la transizione nelle istituzioni letterarie da tipi di testo destinati prevalentemente (e talora esclusivamente) alla recitazione a tipi di testo destinati alla lettura, spesso alla lettura commentata, ed ancora il passaggio da un tipo di documentazione stereotipa tutta interna alla polis, ad un'altra proiettata verso relazioni vaste e articolate in soluzioni testuali differenziate, furono tutti fattori che incisero profondamente sull'evoluzione della scrittura e sulle scritture e sui modi di farsi del libro e del documento. Ugualmente nel mondo greco-romano dei primi secoli dell'impero l'affermarsi di una società largamente alfabetizzata, mediamente colta ed in larga parte burocratica, e l'insorgere nelle istituzioni letterarie di testi stilisticamente semplificati ad uso di quel nuovo pubblico e delle sue istanze culturali, ma anche sociali e religiose, finirono con il determinare, ancora una volta, conseguenze rilevanti nella storia della scrittura sia greca, con l'abbandono di tutta l'eredità grafica ellenistica (salvo riprese arcaizzanti), sia latina, con il passaggio da un sistema di scrittura ad un altro, così come nella storia del libro e del documento, ove s'ebbero, nel primo caso, la lenta ma irreversible sostituzione del codice al rotolo, e nel secondo il caratterzzarsi di gerarchie di scritture e di strutturazioni «diplomatiche» diversificate per tipi di documento. E il discorso potrebbe continuare con l'età tardoantica...

Nel fare storia della scrittura e storia del libro (e del documento) non si può e non si deve prescindere da queste problematiche sullo sfondo. In ultima analisi -fatto ovvio, ma sul quale riflettere- in ogni epoca e ambiente (comunità, gruppo sociale) la capacità o incapacità di leggere e scrivere sono state sempre capacità o incapacità di leggere e scrivere un testo, dalla sua accezioné massima alla minima. In altre parole, scrittura, libro, documento sono sempre prodotti in funzione di un testo (da fissare, o comunicare, o diffondere, o conservare, o per alcuni o tutti questi motivi insieme), ed è quindi nel porsi dialettico con il testo che la loro storia trova un suo spazio ed un suo senso.

\author{
GUGLIELMO CAVALLO \\ Istituto di Paleografia \\ Facoltà di Lettere. Università di Roma \\ (Italia)
}




\section{APPENDICE}

\section{Alcune riflessioni sull'angolo di scrittura}

Il mio scopo, in questa sede, non è quello di ridefinire l'angolo di scrittura in sede teorica (il che ho tentato io stesso una diecina d'anni fa -Ricerche sulla maiuscola biblica, Firenze 1967, p .4 n. 3- ritenendolo «angolo complementare a quello formato dalla retta passante per le punte dello strumento scrittorio con il rigo di base della scrittura, e avente quest' ultimo elemento in comune», definizione che tuttora conservo); né mio scopo è quello di rimetterne in discussione il principio (sotto questo aspetto mi limito a condividere le riserve, ultimamente avanzate, di Marco Palma, Per una verifica del principio dell' angolo di scrittura, in "Scrittura e civiltà» 2 , 1978, pp. 263-273). Piuttosto vorrei porre una questione di fondo, limitata ma ambiziosa: nella dinamica grafica dei primi cinque secoli dell'impero c'è stato un mutamento dell' angolo di scrittura, da "chiuso" ad "aperto", come vero e proprio spartiacque tecnico? In pratica i documenti cardine sono, si sa, il frammento membranaceo del de bellis Macedonicis e il papiro dell'epitome di Livio, diacronicamente posti l'uno al di qua, l'altro al di là di un spartiacque grafico.

Il mio punto di partenza è un papiro omerico pubblicato molto di recente ad opera di più editori in una raccolta di papiri dell'Odissea -per la maggior parte conservati all'Istituto papirologico «G. Vitelli» di Firenze- curata da Manfredo Manfredi (Papiri dell'Odissea. Seminario papirologico 1977-78, Firenze 1979, pp. 19-46 e tavv. 3-6); e di fondamentale importanza è l'esame papirologico, prima ancora che grafico, del manufatto. Si tratta di un rotolo contenente il libro IV dell'Odissea, PSI Od. 5 (inv. CNR 66+67), di cui rimangono, più o meno mutile, sette colonne; ma quella che qui interessa è la parte finale -indicata dagli editori come frammento $\mathrm{C}$ - costituita da sei kollemata e recante tre colonne di scrittura, di cui la prima mutila e l'ultima vergata da altra mano. Tra tali kollemata si distinguono cinque suture o kolleseis, ma, mentre i primi quattro kollemata e le tre suture che li legano sono propri del rotolo originale, gli ultimi due kollemata si devono ritenere ricavati da altro rotolo, confezionato ma non ancora scritto, formato da kollemata di circa 14 centimetri ciascuno, e da cui si dovette tagliare un kollema e mezzo per sostituire la parte del rotolo recante l'ultima colonna, evidentemente deterioratasi. Fu operato, insomma, un vero e proprio "restauro», eliminando l'estremità danneggiata e mettendo al suo posto una striscia, tutta nuova, di papiro, su cui fu trascritta l'ultima colonna del IV libro dell'Odissea; e che di restauro si trattò si desume sia dalla posizione della quarta kollesis, la quale si trova proprio tra penultima e ultima colonna, vale a dire in un particolare intercolumnio, sia dal tipo 
del materiale papiraceo, diverso per lavorazione e costituito da kollemata meno alti e più larghi, sia dalla circostanza che l'ultima colonna risulta vergata da altra mano, più tarda, per giunta, della prima.

Ma passiamo all'esame più strettamente grafico. Per quanto concerne la mano che ha scritto originariamente il rotolo gli editori parlano di «intenzionale arcaismo» e "aspetto inconsueto della scrittura, che mostra forme di tipo più antico tracciate con chiaroscuro di più recente origine" (p. $20 \mathrm{~s}$.), e ne propongono, quindi, una datazione tra il I e il II secolo d.C. E' possibile che le cose stiano così, ma a me pare debba farsi altro discorso, tanto più che si tratta di una scrittura dal tracciato sciolto e spontaneo, priva di qualsiasi artificio che possa farne sospettare una qualche intenzionale imitazione di modelli più antichi. Se si esamina la morfologia delle lettere -in relazione a quanto si conosce delle articolazioni della scrittura greca di età romana, libraria e non, testimoniate da un largo numero di prodoti- è difficile ritenere la scrittura della prima mano del papiro in esame più tarda dell'inizio del 1 secolo d.C.: alpha e lambda con primo tratto obliquo talora in posizione quasi verticale $\mathrm{o}$ ad andamento ricurvo, epsilon o theta con linea orizzontale fortemente ridotta, zeta di forma arcaica e perciò con asta mediana verticale, kappa con tratto obliquo discendente convesso e ascendente in posizione quasi orizzontale, ypsilon quasi privo di linea verticale e con calice profondo e piuttosto stretto, i grossi "epattements» rivolti verso destra alle estremità inferiori di alcuni tratti, sono tutte caratteristiche che, già dislocate in prodotti diversi della fine del I secolo a.C., si incontrano fino alla fine del I d.C., ma non oltre. Si deve parlare, perciò, non di «intenzionale arcaismo», ma di forme grafiche effettivamente piuttosto antiche. Se si osserva il chiaroscuro, si capiscono le ragioni che hanno indotto gli editori ad abbassare in qualche modo la datazione del papiro: l'angolo di scrittura, infatti, si può calcolare intorno a 75 gradi o poco più, ma non senza qualche variazione suscettibile di metterne in dubbio la fissità del valore (di massimo spessore risultano le aste verticali o quelle tendenti all'andamento verticale, ma pure alcuni tratti obliqui discendenti da sinistra a destra, p. es. in alpha, delta, lambda e kappa); in ogni caso l'angolo di scrittura è esattamente lo stesso (e mostra le medesime fluttuazioni) di quello dell'epitome di Livio. Non mi sembra, tuttavia, che tale angolo di scrittura in prevalenza "aperto" debba indurre ad abbassare la data del papiro omerico; piuttosto è da pensare che all' inizio del I secolo d.C., se non pure da prima, già alla fine del I a.C., si adoperasse anche un algolo aperto.

Osserviamo ora, più da vicino, la seconda mano, quella che ha "restaurato" il rotolo. Si tratta di uno stile di scrittura abbastanza diffuso, caratterizzato da disegno molto calligrafico, forte tendenza alla bilinearità, uso di «empattements" decorativi; tra la lettere si possono notare alpha, lambda e delta con tratti obliqui fortemente divaricati, zeta con trasversa ben delineata ed obliqua, $m y$ con tratto mediano unico ed incurvato fino a toccare il rigo di base. "Nel complesso", come scrivono gli editori, «è una scrittura che presenta caratteristiche vicine a quelle della cosiddetta "maiuscola rotonda" " (la scrittura dell'Omero di Hawara, per intendersi) da assegnare senz'altro, perciò, al II secolo. Quanto al chiaroscuro, esso permette di misurare un angolo di scrittura intorno ai 40-45 gradi. Dunque, la prima mano, più antica e comunque non posteriore all' inizio del I secolo d.C., scrive secondo un angolo 
"aperto", 75 gradi o poco più (pur con certe variazioni), la seconda mano, più tarda di circa un secolo o un secolo e mezzo, scrive secondo un angolo piuttosto "chiuso", 40-45 gradi.

Ma v'è di più. Un papiro di Ercolano ancora inedito, P. Herc. 1491, consente di estendere alla dinamica della scrittura latina quanto si è notato per la greca. Ed invero il reperto, ovviamente non posteriore alla data della distruzione della città campana, il 79 d.C., mostra un angolo di scrittura decisamente "aperto», vicino ai 75 gradi pur se non qualche minima variazione (vd. il mio lavoro I rotoli di Ercolano come prodotti scritti I rotoli di Ercolano come prodotti Scritti. Quattro reflessioni, "Scrittura e Civiltà», 8, 1984, p. 28.

Quale, perciò, il responso dei nostri papiri? Innanzitutto mi sembra che non vi sia stato mai, in alcun momento, uno spartiacque, una cesura, tra angolo di scrittura "chiuso" ed angolo di scrittura "aperto", né che vi sia stato, come pure s'è pensato almeno per la scrittura latina, un qualche passaggio graduale dall'uno all'altro: passaggio prima involontario poi consapevole (G. Petronio Nicolaj, Osservazioni sul canone della capitale libraria romana fra I e III secolo, in Miscellanea in memoria di G. Cencetti, Torino 1973, p. 21 n. 55) o "risultato di successivi tentativi, modificazioni adattamenti» (E. Casamassima ed E. Staraz, Varianti e cambio grafico nella scrittura dei papiri latini, Note paleografiche, in "Scrittura e Civiltà», 1, 1977, p. 74); è da ritenere vi sia stata, invece, una coesistenza di angoli (fatto già prospettato da Eric G. Turner, Greek Manuscripts of the Ancient World cit., p. 27) suscettibile di commistione o di prevalenza tra l'uno e l'altro (donde il dubbio metodico introdotto da Marco Palma sul principio stesso, dopo una verifica effettuata proprio sulla scrittura latina); coesistenza dovuta, prima ancora che a vere e proprie tecniche scrittorie diversificate, a fluttuazione del fenomeno all'interno dello scrivere come prodotto fisico, come attività implicante un continuo e libero variare di movimenti. Ma se -come mostrano i papiri qui presi in esame- nella scrittura latina al pari che nella greca, non v'è stato alcuno spartiacque, alcuno svolgimento, non è possibile, neppure, porre al di qua o al di là di uno steccato semplicisticamente «tecnico» $\mathrm{e}$ "diacronico" determinati fenomeni, siano essi il cambio grafico (o passaggio dalla maiuscola alla minuscola, che dir si voglia, in àmbito latino) o la genesi dell'onciale. Le stesse ragioni invocate per spiegare una presunta «rivoluzione tecnica» («inclinaison du papier», taglio diverso del calamo, posizione della mano o altro) $\mathrm{mi}$ sembrano da ribaltare: soprattutto nel caso di scritture organizzate in un sistema chiuso (stile o canone) si deve ritenere non che sia il fattore tecnico a determinare valori grafici (nel caso specifico i valori d'angolo), ma che siano i valori grafici perseguiti a determinare l'assunzione di questo o quel fattore tecnico; ma anche ove si tratti di scritture correnti o "ufficiali», esigenze di economia, di funzionalità o di funzione grafica possono determinare scelte tecniche. In altri termini, il fattore tecnico assunto poteva essere ora l'uno ora l'altro purché atto ad assecondare -mediante un certo angolo più o meno fisso- un qualche intento grafico individuale, di scuola, di tradizione, di ambito geografico, di epoca: gli scriventi si muovevano entro una gamma di soluzioni possibili del problema grafico, correlate ad esigenze complesse e diverse. Non pare vi sia stata mai in età antica (o medievale) una tecnica obbligata e obbligante per chi scriveva: non si è in grado, perciò, di conoscere 
l'angolo che poteva essere adoperato in una determinata epoca, ma soltanto l'incidenza più o meno alta di probabilità, peraltro non assoluta ma relativa; quando, per una serie di convergenti motivi, s'era costituito un particolare indirizzo didattico, uno stile o un canone, quando si passava, in altre parole, dal piano delle forme e delle strutture essenziali al piano sovrastrutturale dei sistemi chiusi.

Sotto quest'ultimo aspetto la scoperta dell' angolo di scrittura conserva ancora oggi, tutta intera, la sua grandissima portata; ma nel quadro generale del divenire storico della scrittura latina, così come della greca, non v'è stata alcuna rivoluzione o mutamento; v'è stata coesistenza, fluttuazione, prevalenza. Mi sembra perciò di dover porre, ormai, una domanda volutamente provocatoria: il frammento del de bellis Macedonicis non può essere stato scritto più tardi del papiro dell'epitome di Livio?

\section{RÉSUMÉ}

L'auteur étudie l'Histoire de l'Écriture et du Livre pendant l'époque des grecs et des romains, en fournissant aux chercheurs un exhaustif état de la question.

\section{SUMMARY}

The author analyses the History of the Writing and the History of the Book during the Greek and Roman Ages, and so he furnishes the searchers with a complete bibliographical information. 